\title{
Modeling and prediction of turbulence-induced wavefront distortions
}

\author{
Rufus Fraanje $^{a, b}$ and Niek Doelman ${ }^{b}$ \\ ${ }^{a}$ Delft Center for Systems and Control, Delft University of Technology, Delft, The \\ Netherlands \\ ${ }^{b}$ TNO Science and Industry, Delft, The Netherlands
}

\begin{abstract}
In recent years various researchers have proposed an optimal control approach for the rejection of turbulence-induced wavefront distortions in an AO system. The essential element in the design of an optimal controller is the choice for the turbulence model, which predicts the turbulence to compensate for the inherent delay in the AO control loop. In this paper various models as proposed in literature are considered; ranging from first order temporal models to high-order full spatialtemporal models. The various models are analyzed and the resulting 1-step ahead predictors are derived. The performance of the predictors are compared for a von Kármán type of turbulence with frozen flow propagation in and time-varying propagation directions.
\end{abstract}

Keywords: Turbulence modeling, Kalman filter, Autoregressive model, 1-step ahead predictor, optimal control, adaptive optics

\section{INTRODUCTION}

In recent years several researchers have addressed the control performance of an adaptive optics (AO) system and in particular the best achievable control performance. The resultant control approach is often presented as optimal control or linear quadratic Gaussian (LQG) control, in which the controller is designed such that the minimum value of the mean-squared wavefront error is obtained. Various approaches to this control design issues have been presented, e.g., ${ }^{1-3}$

The common philosophy behind these control approaches is to achieve the minimum value of the time-averaged, summed squares of the wavefront errors. In general linear control systems, the optimal controller is determined by a number of system properties, including the dynamic model of the disturbance, the dynamic model of the plant and system noise like measurement noise at the sensor and load noise at the actuator. This paper focuses on the dynamic model of the disturbance and the effects of the model for the optimal AO performance. In designing the optimal AO controller, the disturbance model is utilized for prediction of the distortion over the loop delay horizon. $\operatorname{In}^{1-3}$ as well as ${ }^{4,5}$ various disturbance models and predictors have been proposed. These are based on autoregressive (AR) estimation theory or Kalman filtering.

In this paper we will derive and compare AR and state-space disturbance model approximations for turbulence induced wavefront distortions; this will be done in Sec. 2. Because the turbulence induces a disturbance which spectrum is nonrational, finite order AR and state-space models can only approximate the turbulence induced disturbance. It turns out, however, that low order state-space models provide a rather accurate description of the turbulence. In Sec. 3 the AR and Kalman filter predictors are derived. Sec. 4 compares the AR and Kalman filter predictors for a frozen flow von Kármán turbulence. The effect of variations in the flow propagation direction is studied in detail. Finally, Sec. 5 concludes the paper.

Further author information: (Send correspondence to R. Fraanje)

R. Fraanje: E-mail: p.r.fraanje@tudelft.nl, Telephone: +31 152785189

N.J. Doelman: E-mail: niek.doelman@tno.nl, Telephone: +31 152692410

Adaptive Optics Systems II, edited by Brent L. Ellerbroek, Michael Hart, Norbert Hubin, Peter L. Wizinowich, Proc. of SPIE Vol. 7736,773617 • @ 2010 SPIE · CCC code: 0277-786X/10/\$18 · doi: 10.1117/12.858076 


\section{PARAMETRIC MODELING OF WAVEFRONT PHASE FLUCTUATIONS}

\subsection{Correlation in space and time}

In this paper we will assume that the spatial distribution of the turbulence is described by the von Kármán model and its evolution in time by the frozen flow propagation assumption. Let $\phi(t, x, y)$ be the wavefront phase at time instant $t \in \mathbb{R}$ and position $(x, y) \in \mathbb{R}^{2}$, then in $^{6}$ it is shown that the spatial correlation according to the von Kármán model is given by

$$
\mathrm{E}\left(\phi(t, x, y) \phi\left(t, x-\delta_{x}, y-\delta_{y}\right)\right)=c\left(\frac{2 \pi r}{L_{0}}\right)^{5 / 6} K_{5 / 6}\left(\frac{2 \pi r}{L_{0}}\right), \quad r=\sqrt{\delta_{x}^{2}+\delta_{y}^{2}}
$$

and $c=2^{-5 / 6} \Gamma(11 / 6) \pi^{-8 / 3}(\Gamma(6 / 5) 24 / 5)^{5 / 6}\left(L_{0} / r_{0}\right)^{5 / 3}$, where $L_{0}$ is the outer scale of the turbulence, $r_{0}$ the Fried parameter, and $\mathrm{E}($.$) the expectation operator, \Gamma($.$) the Gamma function and$ $K_{5 / 6}($.$) the modified Bessel function of the third type of order 5 / 6$. The frozen flow assumption defines the propagation in time according to

$$
\phi\left(t+\delta_{t}, x, y\right)=\phi\left(t, x-v_{x} \delta_{t}, y-v_{y} \delta_{t}\right)
$$

where $v_{x}$ and $v_{y}$ are the components of the propagation velocity in the $x$ - and $y$-directions respectively. Then, the correlation in space and time can be obtained by using Eq. (1) and (2) together, which yields

$$
R\left(\delta_{t}, \delta_{x}, \delta_{y}\right):=\mathrm{E}\left(\phi(t, x, y) \phi\left(t-\delta_{t}, x-\delta_{x}, y-\delta_{y}\right)\right)=c\left(\frac{2 \pi r}{L_{0}}\right)^{5 / 6} K_{5 / 6}\left(\frac{2 \pi r}{L_{0}}\right),
$$

where $r=\sqrt{\left(\delta_{x}-v_{x} \delta_{t}\right)^{2}+\left(\delta_{y}-v_{y} \delta_{t}\right)^{2}}$.

Now, for sake of simplicity, suppose the telescope has a square apperture with dimension $D \times D$. We will consider the wavefront phase at discrete time instants $t=i T_{s}$ on a discrete grid $(x, y)=(\Delta / 2+j \Delta, \Delta / 2+k \Delta)$ for $i=0,1,2, \cdots$ and $j, k=0, \cdots, N_{s}-1$ where $N_{s}$ the largest integer less or equal than $D / \Delta$. With some abuse of notation $\phi(t, x, y)$ for $t=i T_{s}$ and $(x, y)=(\Delta / 2+j \Delta, \Delta / 2+k \Delta)$ is denoted by $\phi(i, j, k)$ and

$$
R(i, j, k):=\mathrm{E}(\phi(m, n, p) \phi(m-i, n-j, p-k))
$$

which can be determined from (3).

\subsection{Cholesky approach}

Suppose, that we want to simulate the turbulence $\phi(i, j, k)$ for all time instants $i=0, \cdots, N_{t}-1$ and grid points $j, k=0, \cdots, N_{s}-1$. One possibility is by means of a Cholesky factorization. To this end, all elements $\phi(i, j, k)$ are stored in one big vector stacking over all temporal and spatial grid points

$$
\psi:=\left[\begin{array}{lll}
\varphi(0)^{T} & \cdots & \varphi\left(N_{t}-1\right)^{T}
\end{array}\right] \in \mathbb{R}^{N_{t} N_{s}^{2}},
$$

where $\varphi(i)$ the vector stacking over all spatial grid points at time instant $i$

$$
\varphi(i):=\left[\begin{array}{lllll}
\phi(i, 0,0) & \cdots & \phi\left(i, 0, N_{s}-1\right) & \cdots & \phi\left(i, N_{s}-1, N_{s}-1\right)
\end{array}\right] \in \mathbb{R}^{N_{s}^{2}} .
$$

Let $R_{\psi}:=\mathrm{E}\left(\psi \psi^{T}\right) \in \mathbb{R}^{N_{t} N_{s}^{2} \times N_{t} N_{s}^{2}}$ be the covariance of the vector $\psi$, which can be computed by making repetitive use of (4). Further, let

$$
R_{\psi}=Q_{\psi} Q_{\psi}^{T}
$$


be the Cholesky factorization of $R_{\psi}$, where $Q_{\psi} \in \mathbb{R}^{N_{t} N_{s}^{2} \times N_{t} N_{s}^{2}}$ a lower triangular matrix. Then a realization of $\psi$ is given by:

$$
\psi=Q_{\psi} \eta
$$

where $\eta \in \mathbb{R}^{N_{t} N_{s}^{2}}$ a realization of a zero-mean random variable with unity covariance.

Though this method can be applied for any turbulence vector $\psi$ with given positive definite covariance (7), the method will be computationally very complex for large $N_{t}$ and $N_{s}$ since the computational complexity of the Cholesky factorization scales with $N_{t}^{3} N_{s}^{6}$ and the matrix-vector multiplication (8) scales with $N_{t}^{2} N_{s}^{4}$. Sometimes, the matrix $R_{\psi}$ is sparse or is structured such that the Cholesky factorization in (7) can be evaluated more efficiently, but still the method is computationally rather cumbersome. Since we consider the wavefront phase $\phi(i, j, k)$ on an equidistant grid in time and space, the Cholesky factorization and the matrix-vector multiplication may be performed much more efficiently in the frequency domain by exploiting the Fast Fourier Transform (FFT). However, because of the finite window in time and space, spectral leakage will occur which leads to errors in the simulation of $\psi$.

When the velocity vector $\left(v_{x}, v_{y}\right)$ is such that the propagation of the wavefront phase over one sampling instant is matched to the discrete spatial grid, i.e.,

$$
\left(v_{x}, v_{y}\right) T_{s} / \Delta=(j, k) \in \mathbb{Z}^{2}
$$

then, it is sufficient to generate just a long phase screen and to shift this screen at each sampling instant over $\left(v_{x}, v_{y}\right) T_{s} / \Delta$ grid points in the $x$ - and $y$-direction. The standard case is a very huge or wide rectangular screen for the case $v_{x}$ or $v_{y}$ equals zero, but skew rectangular screens may be possible as well. This approach may reduce the number of elements in $\phi$ by a factor $N_{s} \Delta /\left(v_{x} T_{s}\right)$ such that the Cholesky factorization and the matrix-vector multiplication will scale with $N_{t}^{3} N_{s}^{3}\left(v_{x} T_{s} / \Delta\right)^{3}$ and $N_{t}^{2} N_{s}^{2}\left(v_{x} T_{s} / \Delta\right)^{2}$ respectively, for the case $v_{y}=0$. However, for long simulations $\left(N_{t}\right.$ is large) or large grids $\left(N_{s}\right.$ is large) or high velocities $\left(v_{x}\right.$ is high) this may still be computationally infeasible.

\subsection{AR model approach}

Another approach is to model the propagation of the wavefront phase fluctuations over the aperture of the telescope by means of a finite order autoregressive (AR) or autoregressive moving average (ARMA) model. Using finite order AR and/or ARMA models, the correlation of the turbulence can only be modelled by an approximation due to the nonrationality of the von Kármán spectrum. Let the wavefront phase at the spatial grid over the telescope aperture at discrete time instant $i$ be given by $\varphi(i)$, c.f., eq. (6), then the $p$-th order AR model approximating the turbulence is given by

$$
\varphi(i)=\sum_{j=1}^{p} A_{j} \varphi(i-j)+e(i), \quad i \geq 0
$$

where $\varphi(j)=0$ for $j<0$, and $e(i) \in \mathbb{R}^{N_{s}^{2}}$ is a zero-mean white noise stochastic process with covariance $R_{e}:=\mathrm{E}\left(e(i) e(i)^{T}\right) \in \mathbb{R}^{N_{s}^{2} \times N_{s}^{2}}$ and $A_{j} \in \mathbb{R}^{N_{s}^{2} \times N_{s}^{2}}$ are given by the solution to the following Yule-Walker equations

$$
\underbrace{\left[\begin{array}{lll}
R_{\varphi}(p) & \cdots & R_{\varphi}(1)
\end{array}\right]}_{=R_{p: 1}}=\underbrace{\left[\begin{array}{lll}
A_{p} & \cdots & A_{1}
\end{array}\right]}_{=A_{p: 1}} \underbrace{\left[\begin{array}{ccc}
R_{\varphi}(0) & \cdots & R_{\varphi}(p-1)^{T} \\
\vdots & \ddots & \vdots \\
R_{\varphi}(p-1) & \cdots & R_{\varphi}(0)
\end{array}\right]}_{=R_{p}}
$$

where $R_{\varphi}(i)=\mathrm{E}\left(\varphi(m) \varphi(m-i)^{T}\right)$. The covariance matrix $R_{e}$ is given by

$$
R_{e}=R_{\varphi}(p)-A_{p: 1} R_{p} A_{p: 1}^{T}
$$


Note, that the complexity of solving the Cholesky factorization (7) with complexity $N_{t}^{3} N_{s}^{6}$ is replaced by solving the Yule-Walker equations which complexity scales with $p^{3} N_{s}^{6}$, where $p$ is the order of the AR-model which is usually much smaller than $N_{t}$. To generate $e(i)$ a Cholesky factorization of $R_{e}$ need to be made which complexity scales with $N_{s}^{6}$. Then, (10) for $i=0, \cdots, N_{t}$ can be performed with a computational complexity that scales with $(p+1) N_{t} N_{s}^{4}$.

A even more efficient simulation of the turbulence can be performed if the propagation of the wavefront phase per sampling instant is an integer number of grid points in either the $x$ - or $y$-direction. This will be further discussed in Sec. 2.5.

Also note that because of the equidistant sampling in time and space Eq. (11) is highly structured (the matrix $R_{\psi}$ has a hierarchic (block) Toeplitz structure). This structure can be exploited by FFT based methods e.g., to solve (11) much more efficiently. For sparse systems (11) also iterative sparse matrix techniques, like those based on Conjugate Gradients can be used; see e.g. ${ }^{7}$

\subsection{State-space model approach}

Still the order $p$ of the AR model may be relatively high. The number of memory elements, i.e. the state-dimension of the AR model, is given by $p N_{s}^{2}$ and may not be 'minimal'. Therefore, we also consider the state-space model description, which does not suffer from this drawback, i.e. when the state-space model is controllable and observable its state-dimension is minimal, which is denoted by the McMillan degree. Although the model structure of state-space models comprises AR and ARMA models, finite dimensional state-space models can provide an estimate only of the wavefront phase fluctuations.

The fitting of a state-space noise shaping filter which output is a time series which in approximation has a prescribed correlation coefficients is a standard in time series analysis, see e.g. ${ }^{8} \mathrm{In}^{5}$ this method has been applied to the estimation of von Kármán turbulence models. In $^{9}$ a similar method has been proposed that estimates a state-space model in the basis of a given (cross) power spectral densities, and applied to approximate the Kolmogorov turbulence model. Here, we will sketch the realization of a state-space model given the correlation coefficients as given in. ${ }^{8}$

Consider the following state-space model

$$
\begin{aligned}
x(i+1) & =A x(i)+w(i) \\
y(i) & =C x(i)+v(i)
\end{aligned}
$$

where $x(k) \in \mathbb{R}^{n}$ the state and $n$ the state-dimension, $A \in \mathbb{R}^{n \times n}$ a stability matrix, $C \in \mathbb{R}^{\ell \times n}$ the output matrix, and $w(i)$ and $v(i)$ are zero-mean white noise stochastic processes with covariance

$$
\mathrm{E}\left(\left[\begin{array}{l}
w(i) \\
v(i)
\end{array}\right]\left[\begin{array}{l}
w(i) \\
v(i)
\end{array}\right]^{T}\right)=\left[\begin{array}{cc}
Q & S \\
S^{T} & R
\end{array}\right] .
$$

Then it can be shown by straightforward use of (13),(14) and (15) that, the correlation coefficients $R_{y}(i)=\mathrm{E}\left(y(k) y(k-i)^{T}\right)$ are given by

$$
R_{y}(i)= \begin{cases}C P C^{T}+R, & \text { for } i=0, \\ C A^{i-1}(A P+S), & \text { for } i>0,\end{cases}
$$

and $R_{y}(-i)=R_{y}(i)^{T}$ for all $i$, where $P$ is the (positive definite) solution to the Lyapunov equation

$$
P=A P A^{T}+Q
$$

The correlation coefficients can also be considered as the Markov parameters of the state-space system

$$
\begin{aligned}
\xi(i+1) & =A \xi(i)+G \zeta(i) \\
\eta(i) & =C \xi(i)+R_{y}(0) \zeta(i)
\end{aligned}
$$


where $G=(A P+S)$, such that a state-space realization algorithm, as given in e.g., ${ }^{10}$ can be used to determine the matrices $A, G$ and $C$ from the correlation coefficients $R_{y}(i)$. Then $P$ can be determined by solving the Riccati equation

$$
P=A P A^{T}+\left(G-A P C^{T}\right)\left(R_{y}(0)-C P C^{T}\right)^{-1}\left(G-A P C^{T}\right)^{T}
$$

such that $Q$ can be determined using (17) and $S$ and $R$ can be determined using (16).

Hence, given the correlation coefficients $R_{\psi}(i)$ of the time-series $\varphi(i)$ we may find a state-space realization of order $n$ using this procedure, where $R_{y}(i)$ is replaced by $R_{\psi}(i)$. Let this state-space realization be denoted by

$$
\begin{aligned}
x(i+1) & =A x(i)+w(i) \\
\varphi(i) & =C x(i)+v(i)
\end{aligned}
$$

where the covariance of $w(i)$ and $v(i)$ is given by (15).

The computational complexity of the state-space realization is dominated by a singular value decomposition (SVD) of a Hankel type matrix of dimension $p N_{s}^{2} \times p N_{s}^{2}$ where $p \geq 2 n$ and $2 p$ and determines the number of correlation coefficients, $R_{\psi}(i)$ for $i=0, \cdots, 2 p$, that is taken into account in the realization. The computational complexity of the SVD scales with $p^{3} N_{s}^{6}$, which is of the same order of complexity as the estimation of the AR model in the previous subsection. Also note, that again the Hankel type structure can be exploited to obtain a much more efficient SVD. The complexity of solving the Riccati equation (20) scales with $n^{3}$ and is not critical.

Then, given a state-space realization of order $n$ the computational complexity to simulate $\varphi(i)$ for $i=0, \cdots, N_{t}-1$ scales with $N_{t}\left(n+N_{s}^{2}\right)^{2}$ which can be much more efficient than the AR model provided $n \ll p N_{s}^{2}$, where $p$ the order of the AR model, which is true in general. The state-space model can also be transformed to (observable or controllable) canonical forms which complexity is linear in $n$, but usually at the expense of increased sensitivity to round-off errors. Other efficient implementations that scale linearly with $n$ but are less sensitive to round-off errors are given by the input- and output-normal forms. ${ }^{11}$

\subsection{Matched frozen flow propagation}

As already brought up in Sec. 2.2 and 2.3, the computational complexity can be significantly reduced if the propagation in a single sampling instant matches the spatial grid, i.e. (9) holds true. To illustrate how the estimation of the AR and the state-space models are simplified, we will limit to the case that $v_{y}=0$ and $v_{x}$ is such that

$$
v_{x} T_{s} / \Delta=n_{x}, \quad n_{x} \in \mathbb{N} .
$$

Note, that the general case, for which (9) holds true, can always be transformed to this case by a reindexing of the spatial grid along and perpendicular to the propagation direction. In the case $v_{x}$ satisfies $(23)$ and $v_{y}=0$ each sampling instant the wavefront phase is shifted over $n_{x}$ grid points, c.f. Fig. 1, i.e.,

$$
\phi(i+1, j, k)=\phi\left(i, j, k-n_{x}\right) .
$$

As a consequence, the wavefront phase at $\phi\left(i, j, k+m n_{x}\right)$ for $m=1,2, \ldots$ is equal to $\phi(i-m, j, k)$, and thus when a AR or state-space model is given which models the turbulence over the reduced spatial grid $(j, k) \in\left[0, N_{s}\right] \times\left[0, n_{x}\right]$ is given the $\mathrm{AR}$ or state-space model over the whole grid $(j, k) \in\left[0, N_{s}\right] \times\left[0, N_{s}\right]$ can be easily derived by adding time delays to the model. In this way the complexity of solving the Yule-Walker equations (11) is reduced from $p^{3} N_{s}^{6}$ to $p^{3} N_{s}^{3} n_{x}^{3}$ and the simulation of the AR model is reduced from $(p+1) N_{t} N_{s}^{4}$ to $(p+1) N_{t} N_{s}^{2} n_{x}^{2}$. Similarly, the complexity of determing the state-space realization is reduced from $p^{3} N_{s}^{6}$ to $p^{3} N_{s}^{3} n_{x}^{3}$ and the simulation of the state-space model from $\left(n+N_{s}^{2}\right)^{2}$ to $\left(n+N_{s} n_{x}\right)^{2}$. Since, usually $n_{x} \ll N_{s}$ the computational complexity is significantly reduced. 




Figure 1. Matched frozen flow propagation, each sampling time the wavefront phase is shifted over $n_{x}$ grid points along the $x$-axis.

\section{THE AR AND KALMAN PREDICTORS}

Because of the delay in the wavefront sensor and the controller a predicition of the wavefront phase need to be made, which will be discussed in this section. Suppose the measurements of the wavefront sensor are given by

$$
s(i)=\varphi(i)+v(i)
$$

where $v(i) \in \mathbb{R}^{N_{s}^{2}}$ the measurement noise, which is assumed to be a zero-mean white noise stochastic process with covariance $\mathrm{E}\left(v(i) v(i)^{T}\right)=\sigma_{v}^{2} I_{N_{s}^{2}}$. Generally, the wavefront sensor does not provide direct measurements of the wavefront phase, but a derived quantity, e.g., the slopes of the wavefront in the direction of the $x$ - and $y$-axis. In these cases, (25) is replaced by $s(i)=G \varphi(i)+v(i)$ where $G$ the WFS geometry matrix. There may also be additional dynamics in the WFS, like time delay due to read-out, communication and integration time of the CCD device. Here we neglect, without lack of generality, the influence of the wavefront sensor and focus on the problem of wavefront prediction in the presence of measurement noise. Hence, the problem can be stated as: estimate $\varphi(i+1)$ given the measurements $s(j)$ for $j=0, \cdots, i$ given in (25). As a performance criterion we take the variance of the prediction error, i.e.,

$$
J=\operatorname{tr}\left(\mathrm{E}\left((\varphi(i+1)-\hat{\varphi}(i+1 \mid i))(\varphi(i+1)-\hat{\varphi}(i+1 \mid i))^{T}\right)\right)
$$

where $\operatorname{tr}($.$) denotes the trace operator, and \hat{\varphi}(i+1 \mid i)$ the 1-step ahead prediction given all the past measurements over the complete sensor grid up to time instant $i$.

The solution to this minimum variance prediction problem is given by the following 1-step ahead predictor

$$
\hat{\varphi}(i+1 \mid i)=A_{i} s_{i}(i)
$$

where

$$
s_{i}(i)=\left[\begin{array}{llll}
s(i)^{T}, & s(i-1)^{T}, \quad \cdots & s(0)^{T}
\end{array}\right]^{T}
$$

and $A_{i}$ is obtained from

$$
A_{0}=\tilde{S}_{0} \tilde{P}_{0}
$$

where

$$
\begin{aligned}
\tilde{P}_{0} & =\left(R_{\varphi}(0)+\sigma_{v}^{2} I_{N_{s}^{2}}\right)^{-1} \\
\tilde{S}_{0} & =R_{\varphi}(1)
\end{aligned}
$$


and for $i \geq 1$

$$
A_{i}=\left[\begin{array}{ll}
A_{i-1} & 0
\end{array}\right]+\tilde{E}_{i-1}\left[\begin{array}{ll}
\tilde{K}_{i-1}^{T} & -\tilde{R}_{i-1}^{-1}
\end{array}\right]
$$

and

$$
\begin{aligned}
\tilde{K}_{i-1} & =\tilde{P}_{i-1} \tilde{S}_{i-1} \tilde{R}_{i-1}^{-1} \\
\tilde{R}_{i-1} & =R_{\varphi}(0)+\sigma_{v}^{2} I_{N_{s}^{2}}-\tilde{S}_{i-1}^{T} \tilde{P}_{i-1} \tilde{S}_{i-1} \\
\tilde{E}_{i-1} & =A_{i-1} \tilde{S}_{i-1}-R_{\varphi}(i)^{T} \\
\tilde{P}_{i} & =\left[\begin{array}{cc}
\tilde{P}_{i-1}+\tilde{K}_{i-1} \tilde{R}_{i-1} \tilde{K}_{i-1}^{T} & -\tilde{K}_{i-1} \\
-\tilde{K}_{i-1}^{T} & \tilde{R}_{i-1}^{-1}
\end{array}\right] \\
\tilde{S}_{i} & =\left[\begin{array}{c}
R_{\varphi}(i) \\
\tilde{S}_{i-1}
\end{array}\right]
\end{aligned}
$$

This predictor is readily obtained using linear (recursive) prediction theory. ${ }^{12}$ However, the computational complexity and memory of the predictor in (27) grows unboundedly in time. Only when $E_{i}$ given by (35) satisfies $E_{i}=0$ for all $i>p$, the predictor remains constant for $i>p$ and the predictions are based on the finite data window $\{s(i), \cdots, s(i-p+1)\}$. It can be shown that for Auto-Regressive (AR) processes, indeed $E_{i}=0$ for $i>p$ where $p$ is the order of the AR process. Then the AR- $p$, i.e., AR predictor of order $p$ is given by

$$
\hat{\varphi}(i+1 \mid i)=\hat{A}_{p}\left[\begin{array}{lll}
s(i)^{T} & \cdots & s(i-p+1)^{T}
\end{array}\right]
$$

for $i \geq p-1$, where $\hat{A}_{p}$ is obtained by

$$
R_{p: 1}=\hat{A}_{p}\left(R_{p}+\sigma_{v}^{2} I_{p N_{s}^{2}}\right)
$$

and $R_{p: 1}$ and $R_{p}$ are defined similar to (11).

Now, suppose that the turbulence-induced phase fluctuations are adequately modeled by the state-space model $(21),(22)$, then the minimum variance predictor is given by the Kalman filter, ${ }^{13}$ which is given by

$$
\begin{aligned}
\hat{x}(i+1 \mid i) & =A \hat{x}(i \mid i-1)+K(s(i)-C \hat{x}(i \mid i-1)) \\
\hat{\phi}(i+1 \mid i) & =C \hat{x}(i+1 \mid i)
\end{aligned}
$$

where $K$ the Kalman gain given by

$$
K=\left(A P C^{T}+S\right)\left(C P C^{T}+R+\sigma_{v}^{2} I_{N_{s}^{2}}\right)^{-1}
$$

and $P$ is the positive definite stabilizing solution to the Riccati equation

$$
P=A P A^{T}-\left(A P C^{T}+S\right)\left(C P C^{T}+R+\sigma_{v}^{2} I_{N_{s}^{2}}\right)^{-1}\left(A P C^{T}+S\right)^{T}+Q .
$$

\section{SIMULATION STUDY}

\subsection{AR and state-space turbulence models}

To validate the accuracy of the AR and the state-space modeling approaches as described in Sec. 2.3 and 2.4 a simulation study has been performed on an $N_{s} \times N_{s}=8 \times 8$ elements equidistant spatial grid over a square telescope aperture of $1[\mathrm{~m}] \times 1[\mathrm{~m}]$. The Fried parameter is $r_{0}=0.2[\mathrm{~m}]$ and the outer scale $L_{0}=10[\mathrm{~m}]$. The sampling time was chosen to be $T_{s}=6.25[\mathrm{~ms}]$ such that for the propagation speed $\left(v_{x}, v_{y}\right)=(20,0)[\mathrm{m} / \mathrm{s}]$ of the turbulence matches the spatial grid, and at each sampling time the turbulence is shifted over 1 grid point in the $x$-axis direction. Hence, following the approach of Sec. 2.5, the wavefront phase at the grid points $(j, k)$ for $j=1,2, \cdots, N_{s}-1$ 


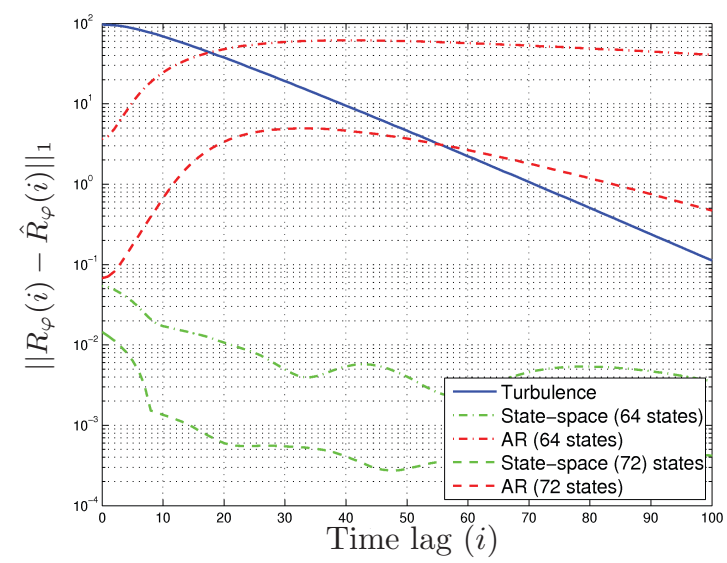

Figure 2. 1-norm of the turbulence correlation coefficients and of the error in the correlation coefficients obtained by AR and state-space models with 62 and 74 states.

can be derived from the grid points at $j=0$ for all $k=0,1, \cdots, N_{s}-1$. Therefore, AR models of order $p=1$ and $p=2$ and state-space models of order $n=8$ and $n=16$ are determined to model the turbulence at $j=0$ for $k=0, \cdots, N-1$. Then, for all the $N_{s}$ grid points along the $y$-axis, $N_{s}-1$ delays are added to model the propagation in time and space, such that in total $N_{s}\left(N_{s}-1\right)=56$ states are added to the model at $j=0$. As a consequence the number of states of the model for $(j, k) \in\left[0, N_{s}-1\right] \times\left[0, N_{s}-1\right]$, including the delays, equals 64 and 72 for both the AR model as well as the state-space model.

The correlation coefficients of the output signal of the AR and the state-space models that should approximate $R_{\varphi}(i)$ are determined and denoted by $\hat{R}_{\varphi}(i)$ for $i=0,1, \cdots, 100$. Fig. 2 shows $\left\|R_{\varphi}(i)\right\|_{1}$, i.e. the summation of the absolute values of all elements of $R_{\varphi}(i)$ (solid line) as well as the 1-norm of the errors in the correlation coefficients $\left\|R_{\varphi}(i)-\hat{R}_{\varphi}(i)\right\|_{1}$ obtained for the AR models and the state-space models with 64 and 72 states.

We observe, that the accuracy of the AR model is increasing with increasing order, but also that the state-space models are a much better approximating the correlation coefficients with the same complexity. The reason is that the model class of the state-space models also comprises moving average filters, and thus is a more general description than the AR model.

\subsection{AR and Kalman filter prediction}

We also computed the AR-1 (the order $p=1$ ) predictor and the Kalman filter on the basis of the state-space model with 64 states. The variance of the noise, $\sigma_{v}^{2}$, has been set such that signal to noise ratios (SNR's) between 0 and $60 \mathrm{~dB}$ are obtained. For comparison, we also computed the prediction errors obtained by: the diagonal AR-1 predictor,

$$
\hat{\varphi}_{j}(i+1 \mid i)=\frac{R_{j, j}(1)}{R_{j, j}(0)+\sigma_{v}^{2}} s_{j}(i), \quad \text { for } j=0, \cdots, N_{s}^{2}-1
$$

where the prediction for each element of $\varphi(i)$ is made independent of information from the other elements, and also the the AR-1 smoother, i.e.,

$$
\hat{\varphi}(i \mid i)=R_{\varphi}(0)\left(R_{\varphi}(0)+\sigma_{v}^{2} I_{N_{s}^{2}}\right)^{-1} s(i)
$$

so, $\hat{\varphi}(i \mid i)$ is used as an estimation of $\varphi(i+1)$ and finally by just taking the last value, i.e., $\varphi(i)$. The predictors are determined for $r_{0}=0.2[\mathrm{~m}], L_{0}=1[\mathrm{~m}]$ and a propagation velocity of $\left(v_{x}, v_{y}\right)=(20,0)[\mathrm{m} / \mathrm{s}]$, and are evaluated on turbulence with the same velocity. Also, performance evaluation has been done with the velocity vector rotated by $90^{\circ}$ and $180^{\circ}$ compared to the modeled velecity vector. As a performance metric, we used the Strehl ratio as computed by

$$
S=e^{-\bar{\sigma}^{2}}
$$





Figure 3. Prediction error in terms of Strehl versus SNR for various predictors determined from the correlation obtained by a propagation velocity of $(20,0)[\mathrm{m} / \mathrm{s}]$ evaluated on turbulence with a propagation velocity of $(20,0)[\mathrm{m} / \mathrm{s}],(0,20)[\mathrm{m} / \mathrm{s}]\left(90^{\circ}\right.$ rotated $)$ and $(-20,0)[\mathrm{m} / \mathrm{s}]\left(180^{\circ}\right.$ rotated $)$. The outer scale was set to $L_{0}=1[\mathrm{~m}]$.

where $\bar{\sigma}^{2}$ the variance of the wavefront phase averaged over all grid points in space.

Fig. 3 shows the resulting Strehl ratio versus SNR curves obtained by the various predictors. We clearly observe, that the Kalman and the AR-1 predictor significantly enhance the performance when the predictor is based on a proper turbulence model that is consistent with the propagation direction and speed. The reason is, that the original temporal error is relatively large, since the propagation within one sampling time instant is over a distance between two neighboring grid point in space. For higher sampling rates, it may be expected that the performance enhancement with the AR-1 and Kalman predictors are less significant. This suggests that - without considering wavefront sensor effects - the sampling time may be increased without significant performance loss when AR-1 and/or Kalman predictors are used. We also observe, that the AR-1 predictor yields about the same performance as the Kalman predictor, only for low SNR's the performance of the Kalman predictor is better, which can be explained by the fact that the Kalman filter is able to better average the effect of noise.

The AR-1 and the Kalman predictor, however, are also more sensitive to variations in the propagation speed or direction, as is clear from the middle and the right graph in Fig. 3 that show the Strehl obtained for changes in the propagation direction. The performance of the AR-1 smoother, the diagonal AR-1 predictor and taking the last value as a prediction, are not sensitive to propagation direction at all, since they do either not make a prediction, or are not depending on the spatial correlation, which changes with the propagation direction. This means, that they are robust for variation in the propagation direction, at the expense of a (significant) performance loss for the case that the propagation direction is taken into account in the model. Similar results 
have been obtained for turbulence with larger outer scales $L_{0}=10[\mathrm{~m}]$ and $L_{0}=100[\mathrm{~m}]$, though the Strehl ratios are lower because the turbulence for larger outer scales is stronger.

\section{CONCLUSIONS}

In this paper finite order AR and state-space models have been derived that approximate the correlation of the frozen-flow turbulence induced wavefront disturbance. It turned out that even with relative low dimensions of the state, state-space models are able to accurately describe the turbulence induced disturbance. Also the predictors corresponding with the AR and the statespace model structures using noisy measurements, i.e., the AR-1 and the Kalman predictor, have been derived. Validation on a simulation example shows that significant performance enhancement may be achieved by accounting for the spatial and temporal correlation, as in the AR-1 and Kalman predictor. For high SNR's the performance of the AR-1 and the Kalman predictor was almost the same, for low SNR's the performance of the Kalman predictor was better because of its ability to better average the effect of measurement noise. Validation on frozen flow disturbances with different propagation directions showed that the AR-1 and Kalman predictor are sensitive to variation in the propagation direction.

Future research will be on the performance versus computational complexity trade-off that can be achieved for various predictors. Also sparsity in the space-time correlation coefficients will be studied as well as the data-driven tracking of the propagation direction and speed.

\section{REFERENCES}

[1] Le Roux, B., Conan, J.-M., Kulcsár, C., Raynaud, H.-F., Mugnier, L., and Fusco, T., "Optimal control law for classical and multiconjugate adaptive optics," J. Opt. Soc. Am. A 21(7), 1261-1276 (2004).

[2] Looze, D., "Minimum variance control structure for adaptive optics systems," J. Opt. Soc. Am. A 23(3), 603-612 (2006).

[3] Hinnen, K., Verhaegen, M., and Doelman, N., "A data-driven $H_{2}$-optimal control approach for adaptive optics," J. Opt. Soc. Am. A 16(3), 381-395 (2008).

[4] Poyneer, L., Macintosh, B., and Véran, J.-P., "Fourier transform wavefront control with adaptive prediction of the atmosphere," J. Opt. Soc. Am. A 24(9), 2645-2660 (2007).

[5] Beghi, A., Cenedese, A., and Masiero, A., "Stochastic realization approach to the efficient simulation of phase screens," J. Opt. Soc. Am. A 25(2), 515-525 (2008).

[6] Conan, R., Modélisation des effects de l'échelle externe de cohérence spatiale du front d'onde pour l'observation à Haute Résolution Angulaire en Astronomie, $\mathrm{PhD}$ thesis, Université de Nice-Sophia Antipolis (2000).

[7] Vogel, C., "Sparse matrix methods for wavefront reconstruction revisited," Proc. SPIE 5490(60), 1327-1335 (2004).

[8] Van Overschee, P. and De Moor, B., "Subspace algorithms for the stochastic identification problem," Automatica 29(3), 649-660 (1993).

[9] Hinnen, K., Verhaegen, M., and Doelman, N., "Robust spectral factor approximation of discrete-time frequency domain power spectra," Automatica 41(10), 1791-1798 (2005).

[10] Kung, S., Arun, K., and Rao, D. B., "State-space and singular-value decomposition-based approximation methods for the harmonic retrieval problem," J. Opt. Soc. Am. 73(12), 17991811 (1983).

[11] Fraanje, R., Robust and fast schemes in broadband active noise and vibration control, $\mathrm{PhD}$ thesis, University of Twente, Enschede, The Netherlands, [online] http://www.dcsc.tudelft. $\mathrm{nl/ \sim rfraanje/thesis.pdf}(2004)$.

[12] Brockwell, P. and Davies, R., [Time Series. Theory and Methods], Springer, Berlin (1991).

[13] Anderson, B. and Moore, J., [Optimal Filtering], Prentice-Hall (1979). 